\title{
Explaining the link between conscientiousness and forgivingness
}

\author{
Hill, Patrick L ; Allemand, Mathias
}

\begin{abstract}
The current studies tested possible explanations for the link between conscientiousness and forgivingness. Using two Swiss adult samples, we examined three reasons why conscientious people tend to be more dispositionally forgiving. Findings suggest that self-regulation ability served as the best explanation for this relationship. Conscientious individuals tend to be better at self-regulation, which in turn enables them to be more forgiving of others. Moreover, this explanation only holds when discussing the link between forgivingness and conscientiousness, and not with respect to why agreeable and emotionally stable participants are more forgiving. Findings are discussed with respect to the role of regulatory processes in explaining the linkages between broad personality dimensions and forgivingness.
\end{abstract}

DOI: https://doi.org/10.1016/j.jrp.2012.05.007

Posted at the Zurich Open Repository and Archive, University of Zurich ZORA URL: https://doi.org/10.5167/uzh-66384

Journal Article

Originally published at:

Hill, Patrick L; Allemand, Mathias (2012). Explaining the link between conscientiousness and forgivingness. Journal of Research in Personality, 46(5):497-503.

DOI: https://doi.org/10.1016/j.jrp.2012.05.007 
Explaining the Link between Conscientiousness and Forgivingness

Patrick L. Hill

University of Illinois at Urbana-Champaign
Mathias Allemand

University of Zurich

Patrick L. Hill, Department of Psychology, University of Illinois at Urbana-Champaign; Mathias Allemand, Department of Psychology, University of Zurich.

Correspondence concerning this article should be addressed to: Patrick L. Hill, 527 Psychology Building, 603 E. Daniel St., Champaign, IL, 61820. E-mail: phill1@illinois.edu. Phone: 217-244-3133. Fax: 217-244-5876.

Acknowledgments: This study is based on data from a research project funded by a grant (NFP 58; No. 4058-115787) from the Swiss National Science Foundation. The preparation of this manuscript was supported by a visiting scholar short visit grant awarded to the authors from the Swiss National Science Foundation (IZKOZ1-133449). 
Explaining the Link between Conscientiousness and Forgivingness 


\begin{abstract}
The current studies tested possible explanations for the link between conscientiousness and forgivingness. Using two Swiss adult samples, we examined three reasons why conscientious people tend to be more dispositionally forgiving. Findings suggest that self-regulation ability served as the best explanation for this relationship. Conscientious individuals tend to be better at self-regulation, which in turn enables them to be more forgiving of others. Moreover, this explanation only holds when discussing the link between forgivingness and conscientiousness, and not with respect to why agreeable and emotionally stable participants are more forgiving. Findings are discussed with respect to the role of regulatory processes in explaining the linkages between broad personality dimensions and forgivingness.
\end{abstract}

Keywords: Forgivingness, conscientiousness, self-regulation, adulthood 


\section{Explaining the Link between Conscientiousness and Forgivingness}

A relatively recent addition to the long literature on forgiveness is work demonstrating that some individuals are dispositionally prone to forgive others across different situations. Several studies though have now examined the personality trait of forgivingness (e.g., Brown, 2003; Brown \& Phillips, 2005), a disposition that not only promotes social relationships (e.g., Paleari, Regalia, \& Fincham, 2005; Maio, Thomas, Fincham, \& Carnelley, 2008) but also personal well-being (e.g., Hill \& Allemand, 2010; 2011). When evaluating specific traits, such as forgivingness, it is valuable to test how these traits correspond to broader trait domains, in order to help connect research on the specific trait to broader literatures. For instance, it has been wellsubstantiated that forgiving individuals tend to score higher on measures of agreeableness and emotional stability (e.g., Balliet, 2010; Steiner, Allemand, \& McCullough, 2012). While these relations are rather intuitive, meta-analytic research also has noted a significant, albeit modest positive relation between forgivingness and conscientiousness, with an average correlation of .17 (confidence interval: .11 to .22; Balliet, 2010). However, it remains a largely unanswered question as to why forgiving individuals also tend to be more conscientious. And yet, examining this relationship could garner valuable insights into why conscientiousness has been linked to greater social well-being in adulthood both concurrently and longitudinally (e.g., Hill, Turiano, Mroczek, \& Roberts, in press).

The current studies tested three different explanations for the link between conscientiousness and forgivingness, using both a sample of health care professionals, and a large sample of participants across the adult years. First, an aging hypothesis would suggest that these traits become linked as a result of the aging process, given that older individuals tend to be 
both more conscientious and forgiving. Second, a maturation hypothesis would posit that conscientiousness and forgivingness are promoted when individuals enter into adult roles (e.g., marriage, parenthood, employment), and thus the correlation between these traits is due to their shared relationship with maturity. Third, a self-regulation hypothesis would propose that conscientiousness is linked to better self-regulation, and that to forgive others necessitates the ability to regulate one's activities and goals. Therefore, conscientious individuals might be more dispositionally forgiving because of their greater ability for self-regulation. We discuss each of these possibilities in greater detail below. However, it is worth noting that these are not mutually exclusive rationales, but rather that the link between conscientiousness and forgivingness could be due to one or more of these reasons.

\section{The Aging Hypothesis}

While debate exists regarding the magnitude of trait changes in adulthood, it appears that personality traits do show systematic relationships with age. For example, cross-sectional and meta-analytic work suggests that levels of conscientiousness tend to increase throughout adulthood (e.g., Allemand, Zimprich, \& Hendriks, 2008; Roberts, Walton, \& Viechtbauer, 2006). Moreover, in one study, positive relations between age and trait levels were even found when examining observer reports of conscientiousness (e.g., Jackson et al., 2009). While that study found that not all facets of conscientiousness show significant age trends, it is worth noting that those facets likely most relevant to forgivingness, such as self-control and reliability, evidenced some of the larger positive correlations with age among the facets examined.

It then is perhaps unsurprising that older individuals tend to be more forgiving as well (see for a review, Allemand \& Steiner, 2012). Indeed, it is a consistent finding that older individuals tend to report higher levels of dispositional forgiveness (e.g., Allemand, 2008; Girard 
\& Mullet, 1997), and discussing these age differences has become a prominent topic in the forgivingness literature (e.g., Steiner, Allemand, \& McCullough, 2011; 2012). One reason why forgivingness tends to increase with age is that people often become more forgiving when they perceive less time left in their lives (Allemand, 2008). Moreover, older adults' tendency to forgive appears to be less moderated by either social pressures or mood (Girard \& Mullet, 1997; Mullet, Houdbine, Laumonier, \& Girard, 1998); put differently, whether older adults forgive a transgression seems less affected by situational variables.

Both age effects coincide with the general trend for personality traits to exhibit "positive" or adaptive changes across the life course (see e.g., Roberts et al., 2006). Accordingly, the relationship between conscientiousness and forgivingness might result from their similar positive relations with age. Specifically, the aging process engenders higher levels on both traits, and thus may explain the relations between the two traits. A similar, yet alternative hypothesis is that maturation might motivate personality development on socially adaptive traits, which would include conscientiousness and forgivingness.

\section{The Maturation Hypothesis}

Theories of personality development have suggested that individuals tend to gain on those traits that help them adjust to adulthood (e.g., Roberts, Wood, \& Caspi, 2008). Evidence for this viewpoint comes from work demonstrating that levels of personality traits during adulthood often are yoked to commitment to adult roles, such as work, family, and community membership (Lodi-Smith \& Roberts, 2007). For example, their meta-analysis found that individuals who invest more in work tend to be higher on agreeableness, conscientiousness, and emotional stability. Similar results were found with respect to family investments. Such relations are intuitive insofar that being agreeable, conscientious, and emotionally stable should allow one 
better success in the workplace and the home. Moreover, several of these relations hold whether examining demographic markers of social roles (e.g., married or unmarried) or looking at more psychological markers (relationship commitment), although psychological markers often demonstrate somewhat stronger effects. That said, conscientiousness was higher among individuals with demographic markers of family and work investments than those without, which constitute the two roles investigated in our studies below.

It is worth noting then that relations have been evidenced between forgivingness and markers of maturation and adaptive social relations. For example, forgiving individuals tend to score higher on identity commitment during emerging adulthood (Hill, Allemand, \& Burrow, 2010). Moreover, forgivingness tends to correlate positively with social well-being indicators (e.g., Hill \& Allemand, 2010), and forgiving individuals experience better marital (Paleari et al., 2005) and family (Maio et al., 2008) relations. Therefore, forgivingness appears likely to be another trait that not only benefits individuals during the maturation process, but also tends to demonstrate mean level increases during this period.

Accordingly, one can posit that the maturation process may engender a link between conscientiousness and forgivingness. One method for examining this is to test whether the two traits correlate similarly for those who have adopted adult roles (e.g., are employed, are currently married, have children) as for those adults who have not. Put differently, adult role adoption should moderate the link between conscientiousness and forgivingness, if increases on these traits are due to this common source, and thus the relationship between these traits should be stronger amongst those currently engaged in the adult role of interest.

The Self-Regulation Hypothesis 
A final hypothesis worthy of discussion is whether conscientious individuals tend to forgive others more because of their better self-regulation skills. Conscientious individuals tend to exhibit greater impulse control, orderliness, and responsibility (e.g., Roberts, Chernyshenko, Stark, \& Goldberg, 2005), traits indicative of individuals who are better at regulating their daily lives as well as their short- and long-term goals. Moreover, researchers have demonstrated that self-regulation is integral to maintaining positive social relationships (see Fitzsimons \& Finkel, 2010 for a review). For instance, self-regulation skills predict romantic partners' sustained prorelational behaviors, and whether they actually follow through on their promises to one another (Kammrath \& Peetz, 2011; Peetz \& Kammrath, 2011). In addition, trait self-control predicts whether individuals avoid their impulses for revenge, and instead seek a more forgiving path for dealing with conflicts (Balliet, Li, \& Joireman, 2011). Thus, conscientious individuals may experience greater relationship success, because they fair better at regulating their long-term relationship goals at the cost of adhering to immediate motives, such as revenge.

This conception of self-regulation is similar to how it is discussed from a SelectionOptimization-Compensation framework (e.g., Freund \& Baltes, 2002), and in line with recent work on self-control and forgiveness in social dilemma tasks (Balliet et al., 2001). However, this view differs conceptually from the work on emotion regulation and forgiveness (e.g., Allemand, Job, Christen, \& Keller, 2008). Accordingly, while that previous work demonstrated that volitional emotion regulation mediates the link between emotional stability and forgivingness, we anticipated that self-regulation should prove a better explanation when considering why conscientious individuals are dispositionally willing to forgive. Moreover, we should note that conscientiousness is not reducible to self-regulation ability on both conceptual (Roberts et al., 2005; Roberts, Jackson, Fayard, Edmonds, \& Meints, 2009) and empirical grounds. For instance, 
our self-regulation measure was derived from work on life management strategies (Freund \& Baltes, 2002), which demonstrated that, while conscientious individuals exhibited better such skills, life management strategies uniquely predicted adult well-being even when controlling for conscientiousness.

\section{Current Studies}

The aim for the current studies was to test three possible explanations as to why conscientious individuals tend to be more dispositionally forgiving. First, we examined whether this link remained when controlling for the variance explained by the aging process. Second, one might expect the relationship between the traits to be moderated by adult role adoption (e.g., being married, having a job, having children), if these traits become correlated as a result of the maturation process. Third, we tested whether self-regulation ability serves to mediate the link between conscientiousness and forgivingness. Here, we employed mediation analyses primarily for explanatory purposes, rather than trying to suggest a developmental patterning for the variables.

If we find support for an explanation, the next question to ask is whether the explanation is unique to the link between forgivingness and conscientiousness. For example, forgivingness demonstrates consistent relations with agreeableness and emotional stability, which generally are stronger than its link to conscientiousness (see e.g., Balliet, 2010; Steiner et al., 2012). Given that conscientiousness, agreeableness, and emotional stability all are indicative of maturity (e.g., Hogan \& Roberts, 2004), it would be of interest to see whether our results are idiosyncratic to the trait of conscientiousness. 
In Study 1, we tested the three explanatory hypotheses using a sample of health care professionals, a group chosen because it reflects a work context in which high levels of both conscientiousness and forgivingness might be particularly adaptive. In line with past work, we expected conscientious individuals would report greater trait forgiveness. We then examined whether this link was moderated by age or adult role status, as well as if it was mediated by selfregulation. As all participants were currently employed, we focused on marital and parental status as the adult role moderators of interest. Finally, we tested whether any identified explanations for the link between conscientiousness and forgivingness also held for the two Big Five traits most frequently associated with forgivingness: agreeableness and emotional stability.

\section{Method}

\section{Sample and Procedure}

One hundred forty-five participants $\left(80 \%\right.$ female; $M_{\mathrm{age}}=46.9$ years, $\left.S D=12.4\right)$ participated in a study of health care professionals working with older adults, in a setting similar to a nursing home. With respect to educational attainment, $12.6 \%$ reported having a basic education (i.e., primary and secondary school) as the highest level of education, $55.3 \%$ completed high school or equivalent (e.g., vocational school), 25.9\% attended advanced professional school, and $6.3 \%$ completed a university education. Regarding marital status, 32.9\% of the sample was single, $41.8 \%$ were married, $21.2 \%$ were separated or divorced, and $4.1 \%$ were widowed. Most participants reported having children (56.6\%). Participants were asked to complete the paper-and-pencil survey to investigate links between personality, behaviors, and health behaviors in a health care sample.

\section{Measures}


Forgivingness. The Tendency to Forgive Scale (TTF; Brown, 2003) was used to assess individual differences in forgivingness. Participants rated their level of agreement to the fouritem scale using a 7-point Likert-type scale from 1 ("Strongly Disagree") to 7 ("Strongly Agree"). Sample items include "I tend to get over it quickly when someone hurts my feelings" and "When people wrong me, my approach is just to forgive and forget." The alpha reliability for the brief composite measure was $\alpha=.62$.

Big Five Personality Traits. Participants completed the German version of the Big Five Inventory (Rammstedt \& John, 2005; John, Naumann, \& Soto, 2008), which consisted of 45 brief descriptive phrases that are prototypical markers of the Big Five factors of personality. Participants used a 5-point Likert-type scale with responses ranging from 1 ("Disagree strongly") to 5 ("Agree strongly") to indicate how well these phrases described their personality. In our sample, the three Big Five traits of interest demonstrated moderate reliability: Conscientiousness $(\alpha=.74)$, Agreeableness $(\alpha=.66)$, Emotional Stability $(\alpha=.75)$.

Self-Regulation. Self-regulation ability was assessed using a subset of the items developed by Freund and Baltes (2002) for assessing selection, optimization, and compensation strategies. Participants rated their level of agreement to the four-item scale using a 6-point Likert-type scale from 1 ("Strongly Disagree") to 6 ("Strongly Agree"). This generic measure of self-regulation consisted of the following items: "I make every effort to achieve a given goal," "I have set my goals clearly and stick to them," "When it becomes harder for me to get the same results, I keep trying harder until I can do as well as before" and "When I can't do something important the way I did before, I look for a new goal.” The alpha reliability for the brief composite measure was $\alpha=.77$. An adapted version of this measure has been used for the study of self-regulation of health behavior (e.g., Ziegelmann \& Lippke, 2007). 


\section{Analytic Plan}

Prior to testing our hypotheses, we sought to replicate previous work by demonstrating that forgivingness correlates positively with (a) conscientiousness, (b) age, and (c) selfregulation. Our analytic plan then focused on the three hypotheses of interest. First, to test the aging hypothesis, we examined whether conscientiousness and forgivingness remained correlated even when controlling for age. Second, to test the maturation hypothesis, we performed multiple regression tests of moderation, to see whether adult role status interacts with conscientiousness (mean-centered prior to forming the interaction terms) to predict forgivingness. We performed these analyses separately for marital status (coded as -1 for single, separated, or divorced, and 1 for married), and parental status ( -1 for no children, 1 for any children). Third, to test the self-regulation hypothesis, we performed a bootstrapping test of mediation using the approach provided by Preacher and Hayes (2008), using 5,000 bootstrapped samples. This approach examines the significance of the indirect effect of conscientiousness on forgivingness through self-regulation.

In addition, for any supported explanations, we performed the same tests with agreeableness or emotional stability in place of conscientiousness. These additional analyses will help examine whether the hypothesis of interest only serves to explain the link between conscientiousness and forgivingness, or whether it applies to other relevant Big Five traits (agreeableness and emotional stability) as well.

With respect to our coding scheme for adult roles, a few caveats are worth pointing out. Unfortunately, our data files did not permit investigating indicators of adult role investment but only adult role status. While previous work (Lodi-Smith \& Roberts, 2007) suggests that psychological adult role markers (i.e., investment) tend to correlate stronger with traits than 
demographic ones (i.e., status), this work also suggests that demographic adult role markers often still correspond to higher levels of adaptive personality traits. In other words, while it would be preferable to have measures of role investment, that meta-analysis does find that demographic indices, such as marital status, tend to correspond to higher levels of conscientiousness. Therefore, we proceeded with our coding scheme to discern differences between participants with or without the role of interest, but discuss the implications of this decision further in the limitations section.

\section{Results}

\section{Correlational Analysis}

Table 1 presents the correlations, means, and standard deviations for the variables in Studies 1 and 2. First, we replicated previous work by demonstrating significant positive relationships between conscientiousness and forgivingness $(r(145)=.20, p<.05)$, between conscientiousness and self-regulation $(r(144)=.32, p<.05)$, and between forgivingness and selfregulation $(r(145)=.22, p<.05)$. However, age was uncorrelated with either conscientiousness $(r(139)=.02, p>.05)$ or forgivingness $(r(140)=.13, p>.05)$.

\section{Aging Hypothesis}

Despite the lack of relations with age, we still tested whether controlling for age might attenuate the correlation between conscientiousness and forgivingness. However, this partial correlation actually was slightly stronger than the initial relationship $(r(136)=.21, p<.05)$. Therefore, we found little evidence for the aging hypothesis in Study 1, but refrain from making any strong conclusions until Study 2 .

\section{Maturity Hypothesis}


To examine the maturation hypothesis, we performed a series of regressions predicting forgivingness levels from levels of conscientiousness, adult role status, and their interaction. Table 2 presents the results from this series of regressions. In line with a maturity hypothesis, both parental status $(\beta=.23, p<.05)$ and marital status $(\beta=.13)$ had positive effects on forgivingness scores, although the latter failed to reach significance. In both cases, conscientiousness remained a significant predictor of forgivingness scores $(\beta=.17$ for parental status, $\beta=.18$ for martial status; both $p$ 's $<.05)$. However, in neither case did adult role adoption moderate this relationship ( $\beta$ 's of .02 and -.13 respectively, both $p$ 's $<.05)^{1}$. Accordingly, we found little support for the maturity explanation in Study 1. Self-Regulation Hypothesis

Finally, we examined whether self-regulation mediated the link between conscientiousness and forgivingness. With self-regulation in the model, the direct effect of conscientiousness on forgivingness was reduced to non-significance $(b=.34[$ s.e. $=.20], p=$ .09). Moreover, the bootstrapping results further suggest that self-regulation was a significant mediator, as the $95 \%$ bias-corrected and accelerated confidence interval for the indirect effect was estimated at .013 to .348 . In other words, using a bootstrapping approach, we evidenced that self-regulation significantly mediated the link between conscientiousness and forgivingness.

Accordingly, we next examined whether this explanation might hold for other Big Five traits. However, neither agreeableness $(r(144)=.16, p>.05)$ nor emotional stability $(r(144)=$ $.07, p>.05)$ were significantly related to self-regulation. Therefore, the self-regulation hypothesis only appears to describe the relationship between conscientiousness and forgivingness.

\section{Discussion}


In Study 1, we provided initial tests of why conscientious individuals tend to be more forgiving, while again replicating the relationship between these two personality traits. Our results found greater support for the self-regulation hypothesis than for the aging or maturity hypotheses. Moreover, the self-regulation explanation only appears to hold for conscientiousness, and not for other known Big Five associates of forgivingness.

Study 2

While Study 1 provides initial insights into the relationship between conscientiousness and forgivingness, we sought to replicate our findings in a larger, more representative sample of Swiss adults. This larger sample will allow a stronger test of all hypotheses, but most importantly of the aging hypothesis. The Study 1 sample was somewhat unique in failing to demonstrate positive relations between age and either conscientiousness or forgivingness, but this may be due to the relatively small sample and focused age range. Moreover, we extended these initial findings by investigating whether employment status, another adult role, might moderate the relationship between these two personality traits, as the initial sample was homogenous with respect to employment status.

\section{Method}

\section{Sample and Procedure}

Nine hundred sixty-two participants $\left(57 \%\right.$ female; $M_{\text {age }}=52.4$ years, $\left.S D=17.7\right)$ participated in a large-scale survey. With respect to educational attainment, $7.0 \%$ reported having a basic education (i.e., primary and secondary school) as the highest level of education, $39.7 \%$ reported attending high school education or equivalent (e.g., vocational school), $24.4 \%$ completed a degree from a technical school, and 28.9\% attended university. Regarding marital status, $35.3 \%$ participants were single, $45.8 \%$ were married, $12.5 \%$ were either separated or 
divorced, and $6.5 \%$ were widowed. In this sample, the majority of participants was employed $(59.8 \%)$ and had children $(53.8 \%)$.

The sampling procedure included an age-stratified random selection of prospective study participants accomplished by the registration office of the city of Zurich. From each one-year age group (1929 to 1989), we included 66 adults with an equal ratio of men and women, resulting in 4,026 prospective participants. Parts of the questionnaire were items on forgivingness, the Big Five personality traits, and a measure of self-regulation ability. The response rate was $24 \%$. After sending the questionnaire package to prospective participants, the procedure included the deletion of postal addresses due to the protection of privacy. Thus, we were not able to remind participants to fill out the questionnaire. In light of this fact, a response rate of $24 \%$ is respectable, particularly given that all participants were unpaid volunteers.

\section{Measures}

The current sample included the same measures of forgivingness, conscientiousness, agreeableness, emotional stability, and self-regulation as in Study 1. The alpha reliabilities for the current sample were $.67, .77, .69, .83$, and .78 respectively for these measures.

\section{Analytic Plan}

The analytic plan was identical for Study 2, with exception that we now included a moderation test by employment status ( -1 for unemployed, and 1 for employed). Therefore, the maturity hypothesis was now investigated using parental status, marital status, and employment status as possible moderators.

\section{Results}

\section{Correlational Analyses}


As in Study 1, forgivingness correlated positively with conscientiousness $(r(950)=.10)$, age $(r(950)=.22)$, and self-regulation $(r(950)=.14)$, all $p$ 's $<.05$. In addition, conscientiousness now correlated positively with both age $(r(950)=.10)$, and self-regulation $(r(944)=.36)$, both $p ’ \mathrm{~s}<.05$. Aging Hypothesis

Next we tested the aging hypothesis, given that both traits were correlated positively with age. When controlling for age, conscientiousness and forgivingness remained correlated $(r(944)$ $=.08, p<.05)$, and to a relatively similar magnitude. Therefore, we found little support for the hypothesis that these traits are related simply because both traits tend to increase with age.

\section{Maturation Hypothesis}

To examine the maturation hypothesis, we performed a series of regressions predicting forgivingness levels from levels of conscientiousness, adult role status, and their interaction. Table 3 presents the results from this series of regressions. In line with a maturation hypothesis, both parental status $(\beta=.13, p<.05)$ and marital status $(\beta=.12, p<.05)$ were significant positive predictors of forgivingness levels. However, in no case did adult role status moderate the link between conscientiousness and forgivingness, and conscientiousness always maintained a significant effect on forgivingness.

Interestingly, employment status negatively predicted forgivingness levels $(\beta=-.16, p<$ $.05)$, counter to a maturation hypothesis. However, this effect appears to result because our sample included several older, retired individuals, as the correlation between age and job status was strongly negative $(r(955)=-.56, p<.001)$, and as shown above, forgivingness positively correlated with age. Although we did not directly assess retirement status, the statutory retirement age in Switzerland is 65 for men and 64 for women. Using this as a proxy for 
retirement, we reran our moderation analysis with only the participants younger than these benchmarks (i.e., those participants who were not required to retire). In this case, we found no mean difference in forgivingness levels between those without jobs $(M=3.71, S D=1.19, n=$ $104)$ and those currently employed $(M=3.65, S D=1.13, n=528)(t(630)=0.39, d=0.05, p>$ .05). Therefore, being employed does not appear to increase or attenuate levels of forgivingness in our sample.

\section{Self-Regulation Hypothesis}

Finally, we tested whether self-regulation served to explain the relationship between conscientiousness and forgivingness. The indirect effect through self-regulation was significant, as the $95 \%$ bias-corrected and accelerated confidence interval ranged from .033 to .152 .

Moreover, the direct effect of conscientiousness was no longer significant in this model $(b=.11$ [s.e. $=.07], t=1.54, p>.05)$. These results suggest that self-regulation scores serve to fully explain the link between conscientiousness and forgivingness.

As a final step, we examined whether the self-regulation explanation held solely for conscientiousness, or whether it also helps to explain why emotionally stable and agreeable people also tend to be more forgiving. Agreeableness failed to correlate with self-regulation scores $(r(944)=.04, p>.05)$, but emotional stability did demonstrate a significant relation with the mediator $(r(942)=.25, p<.05)$. We thus tested mediation with emotional stability as the predictor, but found that the indirect effect through self-regulation was not significant (95\% CI from -.009 to .045). It thus appears that self-regulation ability helps to explain the link between forgivingness and conscientiousness, but not its links with agreeableness or emotional stability. 
The current studies sought to explain the consistent, yet not immediately intuitive, link between conscientiousness and forgivingness (e.g., Balliet, 2010). In particular, we tested three possible reasons why conscientious individuals tend to be more dispositionally forgiving. First, it does not appear that the two traits are linked as a result of the aging process, as their relationship held when controlling for age. Second, we found little evidence that adult role adoption promoted a relationship between these traits, in that their correlation was largely similar for those participants who had and had not adopted the role of interest. Third, our results do suggest that the link between these traits might be explained by self-regulation skills. Conscientious individuals tend to have better self-regulation strategies, which in turn help them to forgive others rather than impulsively seek revenge.

Demonstrating the mediational pathway for self-regulation provides multiple insights for researchers of both conscientiousness and forgivingness. For one, this result falls in line with claims that conscientious individuals have more adaptive social relationships, at least in part, because they are better at negotiating their short- and long-term relationship goals (Fitzsimons \& Finkel, 2010). To decompose this point further, researchers should examine the link between conscientiousness and forgiveness within different relationship contexts. For instance, selfregulation may only serve as a mediator for individuals committed to long-term relationship goals. In addition, our results point to the possibility that examining different self-regulatory processes may further our understanding of forgivingness. While emotional stability seems to promote forgivingness through volitional emotion regulation (Allemand et al., 2008), conscientiousness apparently predicts forgivingness by goal regulation. Indeed, emotional stability evidenced only inconsistent relations with our measure of self-regulation, and it never served to mediate the link between this Big Five trait and forgivingness. Accordingly, we would 
encourage future research that assesses multiple regulatory abilities in a single study, preferably using both self-report and experimental methods, to gain a greater understanding into how different regulatory processes uniquely or interactively predict dispositional and situational forgiveness.

Moreover, it is interesting that self-regulation failed to mediate the link between forgivingness and agreeableness. This findings, paired with the results of Balliet et al. (2011), point to the notion that forgivingness may result from being a prosocial person or from having greater self-control, but these effects may be somewhat orthogonal in nature. Indeed, Balliet et al. (2011) have suggested a compensatory model of forgiveness, in that being higher on trait prosociality or self-control can compensate for lower scores on the opposite trait, in order to facilitate forgiveness. Our findings support this claim by demonstrating that agreeable people were more likely to forgive, and this effect was largely independent of the influence of selfregulation skills on forgivingness.

A couple supplementary points also are worth noting. First, the correlations between conscientiousness and forgivingness evidenced in the current studies were similar in magnitude to the meta-analytic estimate produced by Balliet (2010). Therefore, our findings continue to support the idea that conscientiousness and forgivingness are correlated in the positive direction, albeit somewhat modestly. The modest nature of this relationship might result from the fact that some facets of conscientiousness (such as self-control and responsibility) may be more indicative of forgiving individuals than others (industriousness and orderliness), a point that should be examined in future research. Second, studies like the current ones provide greater insight into how specific traits correspond to the broader trait domains frequently assessed by personality researchers, which in turn promotes our understanding of both the specific and broad trait of 
interest. For example, studies have noted that conscientiousness tends to positively predict markers of relationship quality and social well-being concurrently and longitudinally (e.g., Asendorpf \& Wilpers, 1998; Hill et al., in press; Roberts \& Bogg, 2004). Thus, one explanation for this effect might be that conscientious individuals are more prone to forgive and forget. While the maturity hypothesis did not help explain why conscientiousness is linked to forgivingness, it is worth noting that our results support the idea that adult role adoption may promote positive development on forgivingness. Indeed, participants who were married or parents had significantly higher levels of forgivingness than unmarried participants without children. Such findings, though cross-sectional in nature, at least suggest that being a forgiving person helps one to manage the social roles encountered when becoming an adult, similar to past findings with conscientiousness, emotional stability, and agreeableness (Lodi-Smith \& Roberts, 2007). Future research though is needed to examine this claim with respect to work status. It was difficult to fully test whether having a job motivates forgivingness, because most unemployed participants in the current sample seemed to be retired. Indeed, the current sample had relatively few participants who were younger than retirement age and yet unemployed. Therefore, it would be beneficial to test this claim with a larger sample of unemployed adults.

Although not the primary intent, these results thus provide some foundation for future research on the development of forgivingness. Future longitudinal studies though are needed to truly examine how the adjustment to adulthood influences forgivingness levels, and in turn, whether reciprocal processes exist. For example, one would anticipate that forgiving individuals are predisposed toward committing to long-term social relationships, either with respect to marriage or children. Research has started to demonstrate the possibility of reciprocal relations 
between personality trait and relationship changes (e.g., Neyer \& Lehnart, 2007), and thus it would be valuable to perform similar studies with respect to forgivingness.

In a related vein, it would be valuable to supplement the current work with longitudinal tests of the hypotheses. For example, a stronger test of the maturation hypothesis would be to examine the link between conscientiousness and forgivingness across adulthood, and test whether this relationship differs before and after entering a role (e.g., work, marriage, etc.). In other words, does adult role investment moderate any correlated changes between conscientiousness and forgivingness? Another issue is the lack of precision for our adult role measures. For example, it would be preferable to have a breakdown of different work categories (i.e., full-time employment, part-time employment, unemployed, retired). In addition, it would be worthwhile to assess psychological markers of adult roles, such as levels of investment in and satisfaction with the adult role of interest. Finally, it would be valuable to supplement these analyses with observer reports of personality, to better account for any self-presentation biases, as well as to examine the relations between forgivingness and specific facets of conscientiousness. Moreover, as our forgivingness measure demonstrated moderate but not strong reliabilities, it would be beneficial to include additional, longer measures of trait forgiveness.

In summary, the current studies provide valuable insights into why conscientious people tend to be more dispositionally forgiving. Both traits evidence similar developmental trajectories, and appear to be influenced by prominent adult achievements, which serve as societal markers of maturity. However, a central theme underlying both traits appears to be self-regulation. Accordingly, these findings also speak to the importance of self-regulation for successful aging, given that this construct appears to underlie two traits known to be adaptive in adulthood. 


\section{Footnotes}

1. We report the effects for marital status as a comparison of those currently married versus those not currently in a committed marital relationship. Additionally, we examined the relationships separately for divorced and single participants, to test whether ever having the role influenced the relationship between conscientiousness and forgivingness. In both studies, the correlation between these traits did not differ significantly between the divorced and single groups. Moreover, in no case were the correlations for these two groups lower than those evidenced for currently married participants, counter to the prediction of a maturity hypothesis. Therefore, for parsimony and increased power to detect an effect, we report only those analyses collapsing across these different non-married groups. 


\section{References}

Allemand, M. (2008). Age differences in forgivingness: The role of future time perspective. Journal of Research in Personality, 42, 1137-1147.

Allemand, M., \& Steiner, M. (2012). Situation-specific forgiveness and dispositional forgiveness: A lifespan developmental perspective. In E. Kahls \& J. Maes (Eds.), Justice psychology and conflict: Theoretical and empirical contributions (pp. 361-375). New York: Springer.

Allemand, M., Job, V., Christen, S., \& Keller, M. (2008). Forgivingness and action orientation. Personality and Individual Differences, 45, 762-766.

Allemand, M., Zimprich, D., \& Hendriks, A. A. J. (2008). Age differences in five personality domains across the life span. Developmental Psychology, 44, 758-770.

Asendorpf, J. B., \& Wilpers, S. (1998). Personality effects on social relationships. Journal of Personality and Social Psychology, 74, 1531-1544.

Balliet, D. (2010). Conscientiousness and forgivingness: A meta-analysis. Personality and Individual Differences, 48, 259-263.

Balliet, D., Li, N. P., \& Joireman, J. (2011). Relating trait self-control and forgiveness within prosocial and proselfs: Compensatory versus synergistic models. Journal of Personality and Social Psychology, 101, 1090-1105.

Brown, R. P. (2003). Measuring individual differences in the tendency to forgive: Construct validity and links with depression. Personality and Social Psychology Bulletin, 29, 759771. 
Brown, R. P., \& Phillips, A. (2005). Letting bygones be bygones: Further evidence for the validity of the tendency to forgive scale. Personality and Individual Differences, 38, 627638.

Fitzsimons, G. M., \& Finkel, E. J. (2010). The effects of self-regulation on social relationships. In K. D. Vohs \& R. F. Baumeister (Eds.), Handbook of self-regulation: Research, theory, and applications ( $2^{\text {nd }}$ ed, pp. 407-421). New York: Guilford.

Freund, A. M., \& Baltes, P. B. (2002). Life-management strategies of selection, optimization, and compensation: Measurement by self-report and construct validity. Journal of Personality and Social Psychology, 82, 642-662.

Girard, M., \& Mullet, E. (1997). Forgiveness in adolescents, young, middle-aged, and older adults. Journal of Adult Development, 4, 209-220.

Hill, P. L., \& Allemand, M. (2010). Forgivingness and adult patterns of individual differences in environmental mastery and personal growth. Journal of Research in Personality, 44, 245 250.

Hill, P. L., \& Allemand, M. (2011). Gratitude, forgivingness, and well-being in adulthood: Tests of moderation and incremental prediction. Journal of Positive Psychology, 16, 397-407.

Hill, P. L., Allemand, M., \& Burrow, A. L. (2010). Identity development and forgivingness: Tests of basic relations and mediational pathways. Personality and Individual Differences, 49, 497-501.

Hill, P. L., Turiano, N. A., Mroczek, D. K., \& Roberts, B. W. (In press). Examining concurrent and longitudinal relations between personality traits and social well-being. To appear in Social Psychological and Personality Science. 
Hogan, R., \& Roberts, B. W. (2004). A socioanalytic model of maturity. Journal of Career Assessment, 12, 207-217.

Jackson, J. J., Bogg, T., Walton, K., Wood, D., Harms, P. D., Lodi-Smith, J. L., \& Roberts, B. W. (2009). Not all conscientiousness scales change alike: A multi-method, multi-sample study of age differences in the facets of conscientiousness. Journal of Personality and Social Psychology, 96, 446-459.

John, O. P., Naumann, L. P., \& Soto, C. J. (2008). Paradigm shift to the integrative Big-Five trait taxonomy: History, measurement, and conceptual issues. In O. P. John, R. W. Robins, \& L. A. Pervin (Eds.), Handbook of personality: Theory and research (3 ${ }^{\text {rd }}$ ed.). New York, NY: Guilford Press.

Kammrath, L., \& Peetz, J. (2011). The limits of love: Predicting immediate versus sustained caring behaviors in close relationships. Journal of Experimental Social Psychology, 47, 411-417.

Lodi-Smith, J. L., \& Roberts, B. W. (2007). Social investment and personality: A meta-analytic analysis of the relationship of personality traits to investment in work, family, religion, and volunteerism. Personality and Social Psychology Review, 11, 68-86.

Maio, G. R., Thomas, G., Fincham, F. D., \& Carnelley, K. B. (2008). Unraveling the causes and consequences of forgiveness in families. Journal of Personality and Social Psychology, 94, 307-319.

Mullet, E., Houdbine, A., Laumonier, S., \& Girard, M. (1998). "Forgivingness": Factor structure in a sample of young, middle-aged, and elderly adults. European Psychologist, 3, 289297. 
Neyer, F. J., \& Lehnart, J. (2007). Relationships matter in personality development: Evidence from an 8-year longitudinal study across young adulthood. Journal of Personality, 75, $535-568$

Paleari, G., Regalia, C., \& Fincham, F. D. (2005). Marital quality, forgiveness, empathy, and rumination: A longitudinal analysis. Personality and Social Psychology Bulletin, 31, 368378.

Peetz, J., \& Kammrath, L. (2011). Only because I love you: Why people make and why they break romantic promises. Journal of Personality and Social Psychology, 100, 887-904.

Preacher, K. J., \& Hayes, A. F. (2008). Asymptotic and resampling strategies for assessing and comparing indirect effects in multiple mediator models. Behavior Research Methods, 40, 879-891.

Rammstedt, B., \& John, O. P. (2005). Kurzversion des Big Five Inventory (BFI-K): Entwicklung und Validierung eines ökonomischen Inventars zur Erfassung der fünf Faktoren der Persönlichkeit. [Short version of the Big Five Inventory (BFI-K): Development and validation of an economic inventory for assessment of the five factors of personality]. Diagnostica, 195-206.

Roberts, B. W., \& Bogg, T. (2004). A longitudinal study of the relationships between conscientiousness and the social-environmental factors and substance-use behaviors that influence health. Journal of Personality, 72, 325-354.

Roberts, B. W., Chernyshenko, O., Stark, S., \& Goldberg, L. (2005). The structure of conscientiousness: An empirical investigation based on seven major personality questionnaires. Personnel Psychology, 58, 103-139. 
Roberts, B. W., Jackson, J. J., Fayard, J. V., Edmonds, G., \& Meints, J. (2009).

Conscientiousness. In M. Leary \& R. Hoyle (Eds.), Handbook of individual differences in social behavior (pp. 369-381). New York, NY: Guilford.

Roberts, B. W., Walton, K. E., \& Viechtbauer, W. (2006). Patterns of mean-level change in personality traits across the life course: A meta-analysis of longitudinal studies. Psychological Bulletin, 132, 1-25.

Roberts, B. W., Wood, D., \& Caspi, A. (2008). The development of personality traits in adulthood. In O. P. John, R. W. Robins, L. A. Pervin (Eds.), Handbook of personality: Theory and research (pp. 375-398). New York, NY: Guilford.

Steiner, M., Allemand, M., \& McCullough, M. E. (2011). Age differences in forgivingness: The role of transgression frequency and intensity. Journal of Research in Personality, 45, $670-678$.

Steiner, M., Allemand, M., \& McCullough, M. E. (2012). Do agreeableness and neuroticism explain age differences in the tendency to forgive others? Personality and Social Psychology Bulletin, 38, 441-453.

Ziegelmann, J. P., \& Lippke, S. (2007). Planning and strategy use in health behavior change: A life span view. International Journal of Behavioral Medicine, 14, 30-39. 
Table 1: Means, standard deviations and correlations between variables of interest in Studies 1 and 2.

\begin{tabular}{llllllll} 
& Study 1 M (s.d.) & Study 2 M (s.d.) & 1 & 2 & 3 & 4 & 5 \\
\hline Conscientiousness (1) & $4.03(0.48)$ & $3.67(0.53)$ & --- & $.20^{*}$ & $.32^{*}$ & $.35^{*}$ & $.32^{*}$ \\
Forgivingness (2) & $3.78(1.11)$ & $3.82(1.13)$ & $.10^{*}$ & --- & $.22^{*}$ & $.28^{*}$ & $.24^{*}$ \\
Self-Regulation (3) & $4.38(0.83)$ & $4.11(0.88)$ & $.36^{*}$ & $.14^{*}$ & --- & .16 & .07 \\
Agreeableness (4) & $3.68(0.47)$ & $3.64(0.47)$ & $.21^{*}$ & $.36^{*}$ & .04 & --- & $.38^{*}$ \\
Emotional Stability (5) & $3.38(0.59)$ & $3.36(0.68)$ & $.33^{*}$ & $.40^{*}$ & $.25^{*}$ & $.34^{*}$ & --- \\
\hline
\end{tabular}

Note: * indicates $p<.05$. Correlations above the diagonal refer to Study 1 , while those below the diagonal refer to Study $2 . N^{\prime}$ 's for Study 1 range from 144 to $145 . n$ 's for Study 2 range from 942 to 954. 
Table 2: Multiple regression tests of adult role status as a moderator of the link between conscientiousness and forgivingness in Study 1.

\begin{tabular}{lrrrr} 
Predictor & B & S.E. & $\beta$ & $\mathrm{t}$ \\
\hline & \multicolumn{5}{c}{ Parental Status as Moderator } \\
Conscientiousness & .40 & .20 & .17 & $2.03^{*}$ \\
Parental Status & .26 & .09 & .23 & $2.81^{*}$ \\
Conscientiousness by Parental Status .04 & .20 & .02 & 0.84 \\
& Marital Status as Moderator & & \\
Conscientiousness & .43 & .20 & .18 & $2.17^{*}$ \\
Marital Status & .14 & .09 & .13 & 1.52 \\
Conscientiousness by Marital Status & -.31 & .20 & -.13 & -1.59
\end{tabular}

Note: * indicates $p<.05$. 
Table 3: Multiple regression tests of adult role status as a moderator of the link between conscientiousness and forgivingness in Study 2.

\begin{tabular}{|c|c|c|c|c|}
\hline Predictor & $\mathrm{B}$ & S.E. & $\beta$ & $\mathrm{t}$ \\
\hline \multicolumn{5}{|c|}{ Job Status as Moderator } \\
\hline Conscientiousness & .23 & .07 & .11 & $3.38 *$ \\
\hline Job Status & -.18 & .04 & -.16 & $-4.87 *$ \\
\hline Conscientiousness by Job Status & -.13 & .07 & -.06 & -1.89 \\
\hline \multicolumn{5}{|c|}{ Marital Status as Moderator } \\
\hline Conscientiousness & .15 & .07 & .07 & $2.19 *$ \\
\hline Marital Status & .13 & .04 & .12 & $3.58^{*}$ \\
\hline Conscientiousness by Marital Status & -.12 & .07 & -.06 & -1.67 \\
\hline \multicolumn{5}{|c|}{ Parental Status as Moderator } \\
\hline Conscientiousness & .19 & .07 & .09 & $2.77^{*}$ \\
\hline Parental Status & .15 & .04 & .13 & $4.03 *$ \\
\hline Conscientiousness by Parental Status & $S-.01$ & .07 & -.01 & -0.17 \\
\hline
\end{tabular}

Note: * indicates $p<.05$. 